\title{
Lire des albums complexes en CP : stratégies d'anticipation et construction des significations
}

\section{Caroline Viriot-Goeldel et Jacques Crinon}

\section{Q OpenEdition \\ 1 Journals}

\section{Édition électronique}

URL : http://journals.openedition.org/pratiques/2133

DOI : $10.4000 /$ pratiques. 2133

ISSN : 2425-2042

Éditeur

Centre de recherche sur les médiations (CREM)

\section{Édition imprimée}

Date de publication : 5 juin 2014

\section{Référence électronique}

Caroline Viriot-Goeldel et Jacques Crinon, «Lire des albums complexes en CP : stratégies

d'anticipation et construction des significations », Pratiques [En ligne], 161-162 | 2014, mis en ligne le 05 juin 2014, consulté le 14 novembre 2019. URL : http://journals.openedition.org/pratiques/2133 ;

DOI : 10.4000/pratiques.2133

Ce document a été généré automatiquement le 14 novembre 2019.

(c) Tous droits réservés 


\title{
Lire des albums complexes en CP : stratégies d'anticipation et construction des significations
}

\author{
Caroline Viriot-Goeldel et Jacques Crinon
}

\section{Objectifs et questions}

1 L'échec d'un trop grand nombre d'élèves dans l'apprentissage de la lecture-écriture, attesté par les évaluations internationales (Mullis, Martin, Foy \& Drucker, 2012 ; OCDE, 2011) constitue un défi majeur pour les systèmes éducatifs et notamment pour le système français dont les résultats sont particulièrement médiocres et où l'influence des inégalités socioéconomiques sur les compétences des élèves est particulièrement lourde. Ces échecs ont été mis en relation avec les conditions de socialisation langagière au sein de la famille (Bernstein, 2007 ; Hart \& Risley, 2003 ; Lahire, 2008) : tous les élèves n'arrivent pas à l'école avec les mêmes ressources linguistiques et langagières, le même rapport au langage et les mêmes dispositions à l'étude. La proximité des pratiques familiales avec la socialisation scripturale scolaire semble très liée à la réussite des élèves. L'école, loin de compenser ces inégalités, semble les aggraver par une série de processus que notre équipe de recherche a tenté de comprendre (Bautier \& Rayou, 2009; Rochex \& Crinon, 2011) : l'opacité et le caractère implicite des attendus scolaires, des modes de fonctionnement du système éducatif et des modes de travail exigés des élèves est au cœur des processus d'installation de l'échec scolaire chez les élèves des milieux populaires. À l'inverse, et pour s'en tenir à l'acquisition des compétences de lecteur, les manières d'enseigner qui visent au développement de stratégies explicites de compréhension des textes et au développement d'un retour métacognitif sur l'activité de lecture permettent de réduire les échecs (Goigoux, 2011 ; Palincsar \& Brown, 1984 ; Rémond, 2006).

2 À cet égard, le choix et la manière d'utiliser les supports d'apprentissage jouent un rôle particulier, qui retient plus particulièrement notre attention ici. Ces dernières années 
ont vu se dessiner une évolution notable des supports utilisés pour enseigner la lecture au début de l'école élémentaire. En complément des manuels de lecture, les enseignants français ont régulièrement recours à des supports plus complexes, notamment à des albums de littérature de jeunesse (Quet, 2009; Bonnéry 2010). Cette tendance a été encouragée par un dogme largement diffusé, selon lequel la confrontation précoce avec des textes littéraires complexes permettrait de développer les compétences de compréhension (Tauveron, 1999). La notion de "textes résistants", des textes complexes dont la signification n'est pas immédiatement accessible, qui nécessitent de procéder à de nombreuses inférences ou admettent différentes interprétations, est maintenant largement répandue et il est désormais communément admis que leur étude à l'école devrait commencer le plus tôt possible. L'apparition de la notion de « littérature » dans les programmes de l'école primaire, en 2002, a institutionnalisé le phénomène. En 2007, le ministère français de l'Éducation nationale a publié une liste d'œuvres dont l'étude est recommandée au cycle 2, composée d'une grande variété de genres - de l'imagier à la poésie - présentant des niveaux de difficulté variés. Cette liste comporte de nombreux ouvrages récents qui illustrent parfaitement cette tendance à la complexité analysée, entre autres, par A. Leclaire-Halté (2004), F. Quet (2009) et S. Bonnéry (2010). Ces textes peuvent être caractérisés par une polyphonie narrative, par la présence d'intertextualité, de fins ouvertes, de lacunes volontaires, d'indices ambigus, par le jeu sur les symboles et sur la polysémie, ou encore par une transgression délibérée des caractéristiques des genres littéraires. Ils n'offrent plus au lecteur un chemin linéaire qu'il lui suffirait de suivre, comme le faisaient les livres pour enfants traditionnels (Goldstone, 2002). Certains d'entre eux requièrent également des apprentis-lecteurs certaines connaissances culturelles (Bonnéry, 2010). Ces évolutions des livres pour enfants, et en particulier des albums, ont également été constatées en Amérique du Nord. L'expression «radical change» d'E. T. Dresang $(1999,2008)$ fait référence à ces nouvelles caractéristiques de la littérature enfantine contemporaine, telles l'évolution des formats, les changements de perspective et l'évolution des contraintes (personnages représentés de manières nouvelles et complexes, mises en scènes nouvelles et inhabituelles, fins indéterminées etc.). Or, cette généralisation de l'utilisation de textes complexes dès le début de l'apprentissage de la lecture est fondée sur des intuitions, sur des convictions, plutôt que sur des résultats de la recherche scientifique.

3 La présente recherche entend investiguer les effets de l'utilisation de textes complexes au cours préparatoire $(\mathrm{CP})$ dans un contexte socioculturel défavorisé. Elle examine l'influence combinée des supports choisis et des manières de faire de l'enseignant sur la compréhension des élèves.

\section{Méthodologie}

4 Deux séquences de travail mises en œuvre dans une classe de cours préparatoire d'une école de banlieue seront ici analysées. Située en zone urbaine sensible, cette école est fréquentée par une majorité d'enfants issus de milieux populaires, $75 \%$ des élèves qu'elle accueille appartiennent à des familles de milieux défavorisés. L'enseignante de la classe est expérimentée : maitre formatrice, elle possède 18 années d'ancienneté, dont 13 en cours préparatoire. 
Ces séquences sont issues d'un corpus recueilli dans le cadre d'une étude plus vaste portant sur l'utilisation de textes complexes et composites au cycle 2 de l'école primaire, étude menée par une équipe d'enseignants-chercheurs de notre laboratoire (voir Bautier, Crinon, Delarue-Breton \& Marin, 2012 ; Delarue-Breton \& Bautier, 2013). Chacune de ces deux séquences a été consacrée à la lecture compréhension d'un album. Nous y étudierons l'impact combiné du choix des supports (les albums choisis et lus en classe) et des pratiques de l'enseignante (comment celle-ci conduit les interactions dans la classe et étaye la compréhension de l'histoire) sur la compréhension de ces histoires par les enfants.

Le premier album a été lu et étudié au cours de 14 séances réparties sur trois semaines du mois de décembre, séances d'une durée totale de près de 12 heures, tandis que le second a fait l'objet de six séances de travail réparties sur deux semaines du mois de mai, d'une durée totale de près de sept heures. Cette lecture se faisait parallèlement à la progression liée à l'utilisation d'un manuel. L'ensemble des séances de classe consacrées aux deux albums a été enregistré et retranscrit. Les transcriptions constituent un premier corpus, qui permet de décrire les pratiques de l'enseignante et l'activité des élèves en réponse aux consignes de travail qui leur sont proposées.

7 Un second corpus est composé des produits de l'activité des élèves, qui permettent d'évaluer leur compréhension des albums lus : (i) productions d'écrits intégrées par l'enseignante à sa séquence [voir annexe A] ; (ii) rappel oral guidé par le chercheur, en situation individuelle, après la fin de chaque séquence [voir annexe $\mathrm{B}$ ].

Dans cet article, nous examinerons les supports de lecture choisis par l'enseignante, pour en montrer les difficultés spécifiques, puis le déroulement des deux séquences de travail en mettant en lumière quelques caractéristiques des manières de procéder de l'enseignante - appel à l'émission d'hypothèses par les élèves et pratique de la discussion ouverte-, et enfin les performances effectives des élèves dans les productions que nous utilisons pour évaluer la compréhension. En fin de compte, et pour chacun des albums, la plupart des élèves de cette classe échouent à construire un modèle mental de la situation décrite par le texte compatible avec les données de l'album. Nous tenterons pour finir d'interpréter cet échec. À la difficulté d'un support particulièrement exigeant - contrairement à ce que croient beaucoup d'enseignants -, vient s'ajouter la difficulté pour les élèves à réorganiser par la suite une représentation mentale erronée de la situation décrite par l'histoire, élaborée lors des phases d'émission d'hypothèses. Cette conjonction semble empêcher la plupart de ces élèves aux compétences fragiles de construire des significations et peut-être, de manière plus grave pour la suite de l'apprentissage, de clarifier la nature de l'activité de compréhension de texte et de la compréhension d'albums.

\section{Analyse des supports}

9 Le premier album, Le Bonnet rouge ${ }^{1}$, est inspiré par un texte oral traditionnel. Il appartient à ce genre à structure cumulative que les folkloristes désignent sous le nom de « randonnée ». Un lutin perd son bonnet rouge. Une grenouille s'y installe, bientôt rejointe par une souris, un lapin, un hérisson, un oiseau, un renard, un sanglier, un loup, un ours. À chaque étape est répétée la même ritournelle, dont le charme repose sur des effets de rythme et de rimes : «Tiens, tiens, on dirait que ça bouge, au fond du bonnet rouge... Il y a quelqu'un? Aussitôt la grenouille, la souris... pointèrent le bout du 
nez: Oui ! Oui !... On est ici. Viens donc aussi! » À la fin, il sont serrés au fond du bonnet, «très très serrés ». Et quand survient une... puce, tous les animaux quittent le bonnet en s'écriant « Non! Non !... » Et la puce s'installe dans le bonnet.

Fantaisie de cette histoire absurde et dénuée de réalisme où seule compte l'accumulation d'animaux de plus en plus gros, jusqu'à l'effet comique de la fin, lorsqu'un animal minuscule provoque la fuite des autres. Comprendre ce texte, c'est d'abord se laisser porter par cette progression. Cela suppose - et en même temps aide à construire - chez l'enfant une compétence culturelle, la reconnaissance d'une structure commune à beaucoup de randonnées².

11 En outre, ici, la randonnée a pour support un album, où l'image prend une place importante. Celle-ci, d'ailleurs esthétiquement réussie, n'est pas redondante avec le texte, elle s'en démarque. À chaque page, le dessin foisonne d'animaux (divers insectes) absents du texte et qui ne jouent donc aucun rôle dans l'histoire : autant de sources d'éparpillement possible de l'attention. Le dessin ne respecte pas non plus l'échelle des animaux, brouillant la progression des petits aux gros animaux. Le brouillage que l'image est susceptible d'apporter est d'autant plus important à prendre en considération que l'enseignante, nous le verrons, s'appuie d'abord sur les images pour aborder les albums dans sa classe.

Dans $L a$ recette de $\mathrm{moi}^{3}$, c'est le texte lui-même qui se dérobe à la compréhension. Les images ne brouillent pas le sens, mais n'offrent guère d'indices susceptibles de l'éclairer. Le titre pourrait donner d'emblée une indication : c'est une recette. De quoi ? D'un plat? Un gâteau peut-être ? Non : « de moi ». Qui est ce « moi »? La première page nous informe, mais n'en est pas moins troublante : «Le hasard, lit-on, a fait sa cuisine, neuf mois avant ma naissance ». " Moi » est un narrateur enfant, qui évoque sa propre naissance. Mais qui est ce cuisinier, le hasard ? Personnification d'une notion abstraite, figure de rhétorique de l'allégorie, avec laquelle des enfants de six ans ne sont pas de plain-pied. Et pourquoi neuf mois? Des connaissances sur le monde sont ici requises.

13 Cependant, si "cuisine " est venu confirmer la piste de la recette de cuisine, la difficulté reste entière. Car l'image concrète des activités culinaires auxquelles l'expérience des élèves leur permet de se référer n'est pas suffisante ici. Dans l'album, « cuisine » est une métaphore. Toute la suite repose sur cette métaphore. Tous les traits physiques et moraux hérités par la jeune narratrice - des «boucles de maman » au « sourire de pépé » et aux "grains de colère » qui la conduisent à se disputer avec sa cousine - sont comme les ingrédients de la recette qui a fait de la narratrice ce qu'elle est. Et, conclut-elle à la dernière page, «n'essayez pas de suivre cette recette, elle ne servira plus, je suis unique.»

14 Pour comprendre l'album, il est donc indispensable d'avoir identifié le caractère métaphorique de « recette » et de rapporter toute la suite à ce qui est dit à la première page: «Le hasard a fait sa cuisine». Si la perspective unifiante apportée par la compréhension de la métaphore manque, le reste n'est plus pour le lecteur qu'une suite anecdotique de détails que rien ne vient relier entre eux ; le lecteur échoue à construire une représentation mentale d'ensemble de la situation. 


\section{Influence de la pratique de l'enseignant sur la compréhension des élèves} diverses informations, telles le titre, les sous-titres, les images, les schémas, ou encore des connaissances personnelles. Mises en lien, ces différentes informations peuvent lui permettre d'appréhender la nature ou la thématique d'un texte, d'en envisager le déroulement ou encore le dénouement. Cette stratégie d'anticipation favoriserait l'implication active des élèves dans la lecture et susciterait leur intérêt. En Amérique du Nord, le comité de prévention des difficultés de lecture - Committee on the Prevention of Reading Difficulties in Young Children - recommande ainsi que les programmes des trois premières années d'école élémentaire comportent des émissions d'hypothèses sur les évènements et le dénouement du texte (Snow \& Burns, 1998). Le rapport d'un panel d'experts en lecture, The National Reading Panel Report, mentionne également l'enseignement de l'anticipation, combinée avec d'autres stratégies telles la génération de questions, le résumé ou l'explicitation. Selon ce dernier, ce type d'enseignement présente des résultats mitigés pour l'équivalent du cycle 3 et des effets positifs significatifs pour les niveaux de classe de cinquième et de quatrième au collège (NICHID, 2000 : 4-46). Les programmes d'enseignement français recommandent quant à eux la pratique régulière de l'anticipation des évènements et du dénouement d'un texte à l'école élémentaire (MEN/DGESCO, 2008).

17 Lors des séances d'enseignement de la lecture observées dans le cadre de cette étude, l'enseignante sollicite régulièrement l'émission d'hypothèses.

18 Lorsque l'enseignante introduit en classe La recette de moi, elle distribue un exemplaire de l'album à chacun et invite les élèves à le feuilleter afin d'émettre des hypothèses sur son contenu :

« Vous allez pouvoir, tranquillement, le feuilleter, le regarder, lire, voir ce qu'il y a, dans ce livre. D'accord? Quand vous aurez terminé, je vous donnerai une feuille à chacun et puis vous m'écrirez quelques phrases pour me dire quelle histoire ça nous raconte cet album. Qu'est-ce que vous pensez qu'on va trouver dedans? Et quand on aura terminé, je ramasserai les feuilles. »

«La consigne, je la rappelle: en 1, 2 ou 3 phrases, selon les enfants, vous allez expliquer ce que va nous raconter cet album. Qu'est-ce qu'on va nous raconter comme histoire dans cet album? Ce que vous avez compris en regardant le livre. »

19 La présence du mot « recette » dans le titre et l'illustration d'un gâteau d'anniversaire fait naitre chez plusieurs enfants l'idée d'une vraie recette, parfois de la recette d'un gâteau: «Le bébé prent la recette de sa maman de le gâteaux » (Romane); "C'est l'histoire de la Madame qui fait à manger au Monsieur » (Mehdi); «Des œufs, de la farines et je mélanje » (Abdel) « Des œufs, du riz, du poule, du lait » (Lounes).

Au cours d'un débat d'une durée de 26 minutes, l'enseignante tente d'élucider le titre du livre ainsi que sa première phrase. Cet échange est caractérisé par une succession de malentendus, auxquels vient s'ajouter l'ambigüité linguistique portée par le titre «la recette de moi », expression enfantine pouvant être comprise comme une recette d'un 
plat ou d'un gâteau que possède la narratrice, ou encore comme «la recette pour fabriquer la petite fille", soit une métaphore de la conception de la petite fille par ses parents. Au cours du débat, la réponse «la recette d'une petite fille » est acceptée par l'enseignante, sans pour autant dissiper l'ambiguïté :

M : « Alors Yann... Qu'est-ce que ça veut dire, « la recette de moi ?

$\mathrm{E}:$ C'est une recette de quelqu'un.

$\mathrm{M}$ : Alors la recette de qui, par exemple?

$\mathrm{E}$ : D'une petite fille.

$M:$ La recette d'une petite fille (...).

M : On la voit là, la petite fille. D'accord ? Alors, la recette d'une petite fille. »

21 La dernière phrase consacrée à ce sujet compare la recette et ses ingrédients à une famille et à ses membres, sans clarifier pour autant le sens du titre :

$\mathrm{M}:$ : Est-ce que vous avez une idée de pourquoi on parle de recette? Quand on fait une recette, les enfants on l'a déjà dit ça. Leila, quand on fait une recette, on a besoin de quoi ?

$\mathrm{E}:$ Des ingrédients.

$\mathrm{M}$ : Des ingrédients, très bien. Et on a besoin de petites choses, des ingrédients. Alors maintenant, si on pense à recette dans une famille, est-ce que qu'on aurait comme ça des ingrédients?

$\mathrm{S}:(\ldots)$

$\mathrm{M}$ : Alors nous on pourrait être une somme d'ingrédients, oui. Mais alors ça voudrait dire quoi, que nous, on est une somme d'ingrédients? Que nous on est des ingrédients?

E : Qu'on est une famille. »

Le débat autour de la signification de la première phrase, "Neuf mois avant ma naissance, le hasard a fait sa cuisine " fait encore une fois apparaitre l'idée d'une recette d'un gâteau d'anniversaire :

$\mathrm{M}$ : «Le hasard a fait sa cuisine 9 mois avant ma naissance », ça voudrait dire quoi alors?

$\mathrm{E}:$ Ils ont fait la cuisine.

M : (...) D'accord, oui. Est-ce que vous avez une idée de pourquoi on parle de recette de cuisine?

$\mathrm{E}$ : Parce que le jour où elle était née c'était le jour de son anniversaire et ils ont préparé le gâteau. »

Si le sens des «neuf mois ", correspondant à la durée de gestation de l'enfant, est explicité, la discussion ne conduit pas à des explications plus approfondies satisfaisantes sur le sens de cet incipit pour le moins énigmatique. Elle ne parvient pas à réfuter l'hypothèse selon laquelle l'album contiendrait une vraie recette de gâteau.

Après six séances consacrées à l'album La recette de moi, plusieurs questions ont été posées aux élèves, parmi lesquelles « De quoi parle ce livre? ? et «Pourquoi ce livre s'appelle la recette de moi ?». Leurs réponses montrent que le malentendu n'a pas été dissipé. Certaines réponses sont ambigües, tandis que d'autres témoignent d'une réelle incompréhension. Sept élèves pensent que le titre du livre signifie que la narratrice réalise une recette de cuisine. Lorsque l'on leur demande de plus amples détails, ils mentionnent un gâteau d'anniversaire (3 élèves), un gâteau (2 élèves), ou la recette d'un anniversaire ( 1 élève). Si trois des quatre élèves qui avaient émis l'hypothèse selon laquelle il était question dans ce livre d'une recette de cuisine croient toujours cela à l'issue des six séances, il apparait également que cette idée s'est répandue dans la classe, puisque trois autres élèves l'ont pour finir également adoptée. 
$\mathrm{M}:$ « De quoi parle ce livre?

E : Ça parle d'une recette. Que c'est une petite fille qui la fait. Elle était dans le ventre de sa maman. La maman, elle a eu un enfant. Elle avait fait un anniversaire. Elle a fait une recette avec sa maman (...).

$\mathrm{M}$ : Pourquoi est-ce que ce livre s'appelle La recette de moi ?

$\mathrm{E}$ : Parce que c'est une petite fille qui fait la recette.

$\mathrm{M}$ : Quel genre de recette elle a fait?

$\mathrm{E}:$ Un gâteau à la crème » (Romane).

$\mathrm{M}:$ « De quoi parle ce livre?

E : De la recette de moi. Ben, c'est l'histoire d'une petite fille et elle fait son anniversaire. Elle a des petits grains de colère. Elle est unique.

$\mathrm{M}$ : Pourquoi est-ce que ce livre s'appelle La recette de moi?

E : Parce que quand elle fête son anniversaire, c'est une petite fille qui fait une recette et qui fête son anniversaire.

$\mathrm{M}$ : Elle fait une recette de quoi?

E : D’un gâteau » (Leïla).

échange collectif à l'oral, échange préparatoire à un temps de production écrite. Bien que l'enseignante invite à de multiples reprises les élèves à envisager d'autres scénarios, l'idée d'un bonnet qui éclate en mille morceaux est soulevée sans relâche. Elle est mentionnée douze fois par onze élèves différents, durant une discussion de 21 minutes. N'obtenant pas d'autres réponses, l'enseignante concentre alors ses efforts sur la production d'une suite à cette explosion du bonnet, passant ainsi de «Est-ce que quelqu'un aurait une autre idée, c'est-à-dire pense que le bonnet pourrait ne pas exploser? ? ( $7^{\mathrm{e}}$ minute) à " on peut être d'accord que le bonnet éclate, ou explose, comme vous voulez. Mais, on ne peut pas s'arrêter là. Ce qui est important, c'est de savoir ce qui va se passer après " ( $9^{\mathrm{e}}$ minute). Après cette discussion collective, un groupe composé de six élèves en difficulté dicte à l'enseignante la suite de l'histoire, au cours de laquelle, bien sûr, le bonnet explose. Les autres élèves écrivent individuellement la suite de l'histoire. Pour 16 élèves au total, le bonnet explose ou éclate, tandis que seuls 4 élèves proposent un autre dénouement.

Le débat qui a suivi la lecture du passage de l'arrivée de la puce aurait pu permettre aux élèves de construire une représentation exacte de l'histoire (séance 11, d'une durée de 45 minutes). $\mathrm{Au}$ cours de cet échange, l'enseignante souligne plusieurs points intéressants de l'album: que représente le carré rouge de l'illustration? Et pourquoi une telle représentation? Pourquoi les insectes ont-ils un télescope ? Pourquoi la puce porte-t-elle un drapeau blanc ? Pourquoi le «nnnnnoooooonnnnn » est-il orthographié ainsi? Et finalement, pourquoi les autres animaux ne veulent-ils pas de la puce dans le bonnet? L'analyse des interactions langagières au sein de la classe met en évidence plusieurs caractéristiques de la façon dont l'enseignante conduit la discussion.

La plupart du temps, l'enseignante ne valide ou n'invalide pas les propositions des élèves : afin d'encourager la participation des élèves, elle accueille toute intervention 
sans émettre aucun jugement de valeur. Ce faisant, elle n'offre à ces derniers aucune indication sur la pertinence de ces suggestions, ne les guide pas pour accepter ou rejeter chacune de ces propositions. Il lui arrive même d'accepter des idées contradictoires, comme en témoigne l'exemple de la puce, venue pour faire la paix aussi bien que la guerre :

$\mathrm{M}$, répétant la proposition d'un élève : «Elle a abandonné le bonnet. C'est pour ça qu'elle met un drapeau blanc.

(...)

M : Je reprends ton idée qu'elle viendrait pour faire la guerre. Et quel genre de guerre?

Élève 2 : Le genre de guerre qui ferait qu'elle aurait le bonnet à lui tout seul.

$\mathrm{M}: \mathrm{Tu}$ penses que la puce voudrait récupérer le bonnet pour elle toute seule.

D'accord, et c'est ça l'histoire de la guerre. D'accord. »

À ce moment-là, les élèves se voient proposer par leurs pairs deux explications contradictoires, sans autre commentaire qu'un «d'accord "signifiant seulement sans doute pour l'enseignante qu'elle a compris la proposition de l'élève. Le plus souvent, les discussions autour des questions posées sont interrompues avant que les interlocuteurs ne parviennent à se mettre d'accord sur une réponse, ou que soit proposée une explication pertinente.

De plus, ces débats portent sur la partie du récit qui a fait l'objet d'anticipations lors de la neuvième séance. Néanmoins, l'enseignante ne fait pas référence à ces anticipations pour les vérifier, les valider ou les invalider.

31 Pour finir, plusieurs perturbations brouillent la discussion, dissimulant les véritables enjeux du récit :

- des interventions sans intérêt. Les élèves font de nombreuses propositions erronées ou peu pertinentes: plus de deux minutes de la discussion sont par exemple consacrées à l'intervention d'un élève qui pense que le bonnet ressemble à un bateau, sans que cet échange aboutisse à construire une signification ;

- des comportements perturbateurs. L'enseignante est obligée d'interrompre le débat douze fois pour mettre fin à des comportements inadéquats ;

- une attention détournée sur d'autres points que la compréhension du texte, comme plusieurs minutes de discussion métalinguistiques sur les points d'interrogation, les guillemets ou les pronoms, qui interrompent la discussion sur l'arrivée de la puce.

32 À la fin des quatorze séances consacrées au Bonnet rouge, nous avons demandé individuellement à chaque élève de nous raconter l'histoire oralement. 13 des 18 rappels de récit mentionnent l'éclatement du bonnet, comme si avoir entendu aussi souvent cette idée et l'avoir reprise dans une production écrite l'avait rendue incontestable aux yeux des élèves, comme en témoignent par exemple les récits de Romane, l'une des meilleures élèves de la classe, et de Samia, l'une des plus faibles :

«Le lutin. Et après, la branche lui piquait le bonnet. Et il s'en rend pas compte. Après, il va et le bonnet resta dans l'herbe. Et après, la grenouille rentra dans le bonnet. Et après une souris demande à la grenouille si elle peut entrer dans le bonnet. Après, un lapin vient au bonnet demande s'il peut rentrer dans le bonnet avec la grenouille et la souris. Après, un hérisson vient et demande : «Est-ce que moi je peux rentrer dans le bonnet? " « Oui ». L'oiseau arriva au bonnet et rentra tout de suite et... euhh... Et demanda s'il peut rentrer dans le bonnet. Le sanglier arriva au bonnet et demande s'il peut rentrer avec les autres. Après, un loup arriva au bonnet et demande s'il peut venir dedans. Après, un renard demande s'il peut rentrer dans le bonnet avec les autres. Et un ours arriva au bonnet et veut rentrer 
dedans et le demande. Après, le bonnet se déchire et tout le monde est tombé " (Romane).

«Et après, on est venu, et après le sanglier, il a dit « Tiens tiens, on dirait que ça bouge au fond du bonnet rouge ». Après, il a fait "ouahhh», et quand il fait " ouaah », après la grenouille, et la souris, et le lapin, et le renard, et l'ours ils ont dit : «Oui, oui, on est ici, viens donc aussi ! ». Et après le sanglier il est rentrée, et quand le sanglier il est rentré, ils commençaient à être serrés. Et quand ils commençaient à être serrés, ensuite, ils commençaient à être serrés, et ensuite, ils ont déchiré le bonnet, ensuite, le lutin il a dit : «Il est où mon bonnet? ". Ensuite, il l'a pendu, il était dans l'herbe, et tous les animaux rendaient le bonnet en mille morceaux à le lutin » (Samia). 
$\mathrm{M}:$ «Tu peux me redire les noms de tous les animaux?

$\mathrm{E}$ : La grenouille, l'hérisson, l'oiseau, et après, le bonnet il est trop serré.

$\mathrm{M}$ : Est-ce qu'il y avait aussi un animal minuscule?

E : C'était la souris?

$\mathrm{M}:$ Non.

$\mathrm{E}:$ C'était l'oiseau?

$\mathrm{M}:$ Non.

E : La puce?

$\mathrm{M}$ : Parle-moi de cette puce!

$\mathrm{E}$ : Elle a bu tout le sang peut-être. Elle a bu tout le sang, je sais pas...

$\mathrm{M}$ : Le sang de qui ?

$\mathrm{E}:$ De tous les animaux » (Lounes).

$\mathrm{M}:$ «Qu'est-ce qui s'est passé avec les puces?

E : Il lui a bu le sang de la tête » (Djamila).

«Mais elle a fait exprès de rentrer. Elle est partie en-dessous de leur jambe, elle leur a tiré leur pantalon, elle leur a bu leur sang » (Ahmed).

\section{Conclusions}

L'enseignante, dans les deux séquences, commence la lecture de l'album de la même façon. Sa démarche consiste à demander aux élèves de regarder le livre de manière globale, soit en s'intéressant à sa couverture (Le Bonnet rouge), soit en le feuilletant ( $L a$ Recette de moi), afin de mettre les élèves en situation d'anticiper la signification du texte. L'enseignante stimule les élèves pour leur demander, dans une séance collective, de dire ce qu'ils pensent avoir compris, à partir de ce qu'ils ont vu et lu. En cela, l'enseignante observée fait comme beaucoup d'autres. Cette manière de commencer une séquence de lecture compréhension est fréquente dans les cours préparatoires français, comme nous avons pu le constater lors de nos fréquentes visites dans les écoles.

L'objectif de conduire les apprentis lecteurs à anticiper n'est pas dénué d'arguments, nous l'avons indiqué plus haut. L'activité d'anticipation contribue en effet à construire une cohérence entre les informations du texte pour aboutir à un "modèle de situation » (Van Dijk \& Kintsch, 1983), entraine à cette construction et peut ainsi aider le jeune lecteur à utiliser des stratégies de lecture efficaces et à réguler sa lecture en remettant en cause la représentation partielle ou inexacte de la situation qu'il s'est d'abord construite. En outre, lorsqu'il s'agit, comme ici, de lire des récits littéraires, anticiper permet de gouter à une certaine forme de plaisir littéraire, en confrontant des attentes à ce que révèle la suite de la lecture : les attentes sont comblées - comme dans les contes ou les romans sentimentaux - ou bien au contraire déçues - comme dans les nouvelles à chute.

Cependant, le problème est qu'ici cette stratégie s'applique à des albums et que les élèves, pour répondre à la demande de l'enseignante, s'appuient surtout sur les images, images qui, on l'a vu, en raison même de la qualité de ces albums, offrent peu de redondance avec le texte et peu d'indices pour leurs hypothèses.

En outre, une deuxième caractéristique de la façon de faire de l'enseignante est qu'elle laisse la discussion ouverte lors de ces premières séances. Elle se garde bien de confirmer ou d'infirmer les propositions des élèves et laisse au contraire toutes les hypothèses s'exprimer. Cette situation est un exemple de ce que notre équipe a décrit dans plusieurs de ses travaux, l'instauration d'un malentendu sociocognitif (Bautier \& 
Goigoux, 2004 ; Bautier \& Rochex, 2007 ; Bonnéry, 2007). Pour les élèves qui, dans leur famille, n'ont pas été initiés aux pratiques légitimes de l'univers scolaire, le sens des situations scolaires peut être complètement différent de celui que leur enseignant a tenté d'y donner, faute d'une explicitation suffisante de l'enjeu et des buts de la situation. L'enseignante veut s'en tenir d'abord à un inventaire des hypothèses émises par les élèves, pour pouvoir les mettre dans un second temps à l'épreuve d'un travail précis de recherche du sens véritable, page après page. Mais la plupart des élèves en retiennent autre chose. Le silence de la maitresse vaut approbation. Si celle-ci ne les reprend pas, c'est, pensent-ils, parce que leurs interventions sont recevables. Ce qui est dit lors de la séance d'anticipation est mémorisé comme "bonne réponse » et repris ensuite, dans les productions écrites et les rappels oraux, non seulement par les auteurs des réponses, mais aussi par d'autres élèves.

Enfin, après la lecture complète des albums et plusieurs échanges sur leurs contenus, l'enseignante ne revient pas sur les anticipations qui ont été faites, afin de permettre leur validation, ou au contraire, de montrer en quoi l'histoire s'est avérée prendre un tour différent de celui que les élèves avaient pressenti. Rien n'est jamais explicité, en fin de compte, de ce qui pourrait rendre la pratique de l'anticipation efficace pour construire et reconstruire les significations de ce qu'on est en train de lire, du va-etvient nécessaire entre des compréhensions provisoires de l'album et la confrontation de celles-ci avec ce que la lecture et la relecture nous apporte comme informations nouvelles. L'attention au texte et à l'image, qui interdirait par exemple, dans Le Bonnet rouge, de penser que le bonnet éclate, n'est pas spontanée, elle ne nait pas de la simple discussion entre élèves et ne s'acquiert pas sans le guidage et l'explicitation de l'enseignant. On observe ici des élèves qui se méprennent sur ce qu'est lire un album, activité qu'ils assimilent à l'invention d'une histoire à partir de quelques éléments de départ. Des recherches consacrées à des lecteurs plus âgés, élèves de collège, mettent en évidence le même malentendu (Bautier \& Rayou, 2013). On constate que celui-ci possède des origines précoces, dès le début de la scolarité.

44 Ainsi, notre étude remet en cause l'enthousiasme de C. Tauveron (1999) de B. P. Goldstone (2002) ou de S. Pantaleo (2004) pour la lecture d'albums complexes au cycle 2. Elle serait, selon eux, l'occasion pour les élèves d'explorer différents niveaux d'interprétation, d'apprendre à faire des inférences et à combler les blancs du texte, ce qui serait une caractéristique de la lecture de la littérature, dans une perspective inspirée par les théories de la réception (Iser, Eco...). Les échanges «ouverts» entre élèves dans la classe permettraient de les impliquer de manière active dans la construction de la signification.

La présente recherche met au contraire en évidence que cette construction des significations n'a rien d'automatique, bien au contraire, pour beaucoup d'élèves, et tout particulièrement pour les élèves en difficulté qui composaient cette classe de zone urbaine sensible. Il apparait que la manière qu'a l'enseignante d'étayer (ou non) la construction de la signification est particulièrement déterminante pour permettre aux jeunes élèves les moins pourvus de culture des albums de comprendre et d'interpréter ceux-ci. Par culture, nous entendons à la fois la familiarité avec les genres et les caractéristiques des albums lus et les stratégies à utiliser, en particulier dans la mise en relation d'informations d'origines multiples: texte, images, connaissances du monde, connaissances des textes. Que l'enseignante connaisse les processus de la compréhension et les habiletés à entrainer ne suffit pas, on le voit ici, dès lors que les 
élèves ne prennent pas conscience de ce qu'on leur demande de faire et que leur activité cognitive réelle diffère de l'activité visée.

Il n'est sans doute pas déraisonnable, pour enseigner la compréhension de textes aux jeunes élèves, de s'appuyer sur des textes offrant certaines caractéristiques qui les rendent difficiles, si cette difficulté n'excède pas les capacités d'élèves de six ans. Car comment enseigner autrement les stratégies permettant de résoudre les problèmes posés? Mais utiliser de tels supports implique d'en prendre en compte les caractéristiques : ainsi les perturbations introduites par l'image dans Le Bonnet rouge ou la valeur résumante de la métaphore de la recette dans La Recette de moi. C'est là sans doute une des difficultés, pour l'enseignant, à concevoir son enseignement de la lecture: il n'est pas de scénario standard, reproductible quel que soit le texte. Il importe que l'enseignant analyse soigneusement les caractéristiques des supports de lecture et qu'il les choisisse en toute conscience des difficultés que les élèves sont susceptibles de rencontrer pour les lire. Concevoir une séquence de lecture, c'est aussi prendre en compte la nature du support, tout particulièrement lorsque l'hétérogénéité sémiotique ou l'éclatement de la narration classique contribue à brouiller les repères des jeunes lecteurs.

\section{BIBLIOGRAPHIE}

BAUTIER, É., CRINON, J., DELARUE-BRETON, C. \& MARIN, B. (2012) : « Les textes composites : des exigences de travail peu enseignées? ", Repères, 45, p. 63-79.

BAUTIER, É. \& GOIGOUX, R. (2004) : « Difficultés d'apprentissage, processus de secondarisation et pratiques enseignantes : une hypothèse relationnelle ", Revue française de pédagogie, 148 , p. 89-100.

BAUTIER, É. \& RAYOU, P. (2009) : Les inégalités d'apprentissage. Programmes, pratiques et malentendus scolaires, Paris, Presses universitaires de France.

BAUTIER, É \& RAYOU, P. (2013) : « La littératie scolaire : exigences et malentendus. Les registres de travail des élèves ", Éducation et Didactique, 7 (2), p. 29-46.

BAUTIER, É. \& ROCHEX, J.-Y. (2007) : « Apprendre : des malentendus qui font la différence », in : J. Deauvieau \& J.-P. Terrail (éds.), Les sociologues, l'école et la transmission des savoirs, Paris, Éd. La Dispute, p. 227-241.

BERNSTEIN, B. (2007 [1996]) : Pédagogie, contrôle symbolique et identité, trad. de l'anglais par G. Ramognino Le Déroff \& P. Vitale, Québec, Presses de l'université Laval.

BONNÉRY, S. (2007) : Comprendre l'échec scolaire, Paris, Éd. La Dispute.

- (2010) : "“- Loup y es-tu ? - pas exactement, c'est pour mieux te faire réfléchir, mon enfant..." Sociologie du lecteur supposé par la littérature de jeunesse ", in : actes du congrès de l'Actualité de la recherche en éducation et en formation (AREF), université de Genève.

CANUT, E. \& VERTALIER, M. (2012) : «Lire des albums : quelle compréhension et quelle appropriation par les élèves de maternelle? », Le Français aujourd'hui, 179, p. 51-65. 
DELARUE-BRETON, C. \& BAUTIER, É. (2013) : « Restitution de récit et nouvelle littératie scolaire. Étude comparée de productions orales d'élèves issus d'établissements scolaires de milieux contrastés », communication au $9^{\mathrm{e}}$ colloque de l'IAMTE, Créteil, juin 2013.

DOYON, D. \& FISHER, C. (dirs) (2010) : Langage et pensée à la maternelle, Québec, Presses de l'université de Québec.

DRESANG, E. T. (1999) : Radical Change: Books for Youth in a Digital Age, New York, H.W. Wilson.

- (2008) : «Radical change Revisited : Dynamic Digital Age Books for Youth », Contemporary Issues in Technology and Teacher Education, 8 (3) (en ligne : http://www.citejournal.org/vol8/iss3/ seminal/article2.cfm).

GoIGoUX, R. (2011) : «Une pédagogie éclectique au service des élèves qui ont le plus besoin de l'école ", La Nouvelle Revue de l'adaptation et de la scolarisation, 52, p. 22-30.

GOLDSTONE, B. P. (2002) : « Whaz Up with Our Books? Changing Picture Book Codes and Teaching Implications », The Reading Teacher, 55 (4), p. 362-370.

HART, B. \& RISLEY, R. T. (2003) : « The Early Catastrophe » American Educator, 27 (1), p. 4-9.

LAHIRE, B. (2008) : La raison scolaire, Rennes, Presses universitaires de Rennes.

LECLAIRE-HALTÉ, A. (2004) : « Un album "polyphonique” au cycle III. Histoire à quatre voix d'Anthony Browne », Pratiques, 123-124, p. 113-142.

Ministère de l'Éducation nationale (MEN)/Direction générale de l'enseignement scolaire (DGESCO) (2008) : Une culture littéraire à l'école. Littérature à l'école. Ressources pour le cycle 3 (en ligne : http://media.eduscol.education.fr/file/ecole/46/9/culture-litteraire-ecole_121469.pdf).

MULLIS, I. V. S., MARTIN, M. O., FOY, P. \& DRUCKER, K. T. (2012) : PIRLS 2011 International Results in Reading, Chestnut Hill, TIMSS \& PIRLS International Study Center.

National Institute of Child Health and Human Development (2000) : Report of the National Reading Panel. Teaching children to read: an evidence-based assessment of the scientific research literature on reading and its implications for reading instruction: Reports of the subgroups (NIH Publication No. 00-4754), Washington, Government Printing Office.

OCDE (2010) : PISA 2009 Results: What Students Know and Can Do - Student Performance in Reading, Mathematics and Science (Volume I) (en ligne : http://www.oecd.org/pisa/pisaproducts/ 48852548.pdf).

PALINSCAR, A. S. \& BROWN, A. L. (1984) : « Reciprocal Teaching of Comprehension-Monitoring Activities », Cognition and Instruction, 1 (2), p. 117-175.

PANTALEO, S. (2004) : Young Children and Radical Change Characteristics in Picture Books, The Reading Teacher, 58 (2), p. 178-187.

QUET, F. (2009) : « Qui résiste aux albums résistants?», in : E. Canut \& A. Leclaire-Halté (éds), "L'élève et la lecture d'albums. Quelle compréhension du texte et des images? ", Diptyque, 17, p. 97-115.

RÉMOND, M. (2006) : « Éclairages des évaluations internationales PIRLS et PISA sur les élèves français ", Revue française de pédagogie, 157, p. 71-84.

ROCHEX, J.-Y. \& CRINON, J. (dirs) (2011) : La construction des inégalités scolaires, Rennes, PUR. SNOW, C., BURNS, S. \& GRIFFIN, P. (éds.) (1998) : Preventing Reading Difficulties in Young Children, Washington, National Academy Press. 
TAUVERON, C. (1999) : « Comprendre et interpréter le littéraire à l'école : du texte réticent au texte proliférant ", Repères, 19, p. 9-38.

VAN DIJK, T. A. \& KINTSCH, W. (1983) : Strategies of discourse comprehension, New York, Academic Press.

\section{ANNEXES}

\section{Annexe A. Tâches de production écrite}

Durant les séances consacrées au Bonnet rouge, les élèves ont été invités à produire quatre écrits différents en relation avec le livre :

- après une description collective de la couverture de l'album, l'enseignante demande aux élèves d'écrire ce qu'ils voient sur la couverture, ce à quoi cela les fait penser, et ce qu'ils pensent qui va se passer dans le livre (séance $n^{\circ} 1$ ) ;

- les élèves sont invités à écrire un début d'histoire similaire, celle d'un bonnet vert dans lequel arrivent d'autres animaux (séance $\mathrm{n}^{\circ} 7$ ) ;

- après la lecture du passage correspondant à l'arrivée de l'ours dans le bonnet, les élèves écrivent la suite de l'histoire en complétant la phrase «ils commençaient à être très serrés tout au fond du bonnet, très très serrés... » (séance $n^{\circ} 9$ ) ;

- après la lecture de la fin de l'histoire du Bonnet rouge, les élèves imaginent la suite de l'histoire, après que le lutin repart avec son bonnet... et la puce dedans (séance $\left.\mathrm{n}^{\circ} 11\right)$.

Les séances consacrées à l'ouvrage La Recette de moi comportent quatre temps d'écriture :

- à la suite d'une discussion collective à propos de la couverture du livre, les élèves sont invités à écrire quelques phrases anticipant sur le contenu de l'album (séance $\left.\mathrm{n}^{\circ} 1\right)$;

- après une discussion sur les ressemblances physiques de la narratrice avec les membres de sa famille, les élèves décrivent cette dernière au moyen d'un organisateur graphique, puis écrivent une ou deux phrases pour dresser son portrait (séance 3) ;

- les élèves écrivent le portrait d'une petite fille en complétant un texte à trous en utilisant les mots d'une liste (séance $\left.n^{\circ} 4\right)$;

- les élèves dressent leur portrait en remplissant un texte à trous (séance $n^{\circ} 6$ ).

\section{Annexe B. Procédures d'évaluation}

\section{Le Bonnet rouge}

La compréhension du récit a été évaluée par un rappel de récit, suscité par la question suivante : « Pourrais-tu me raconter l'histoire du Bonnet rouge?». Durant le récit de 
l'élève, le chercheur a pu être amené, le cas échéant, à poser différentes questions et à proposer différents étayages :

- encouragements, invitation à continuer (oui, très bien, et que se passe-t-il après ?) ;

- validations ;

- répétitions, récapitulations ;

- demandes de précisions (qu'est-ce que tu veux dire par... ?) ;

- demandes d'explicitations (Pourquoi ? Comment? Où ?).

\section{La Recette de moi}

La compréhension de l'histoire a été évaluée par un rappel de récit suivi de quatre questions :

- Pourquoi est-ce que ce livre s'appelle La Recette de moi ?

- Je te lis la première phrase : «9 mois avant ma naissance, le hasard a fait sa cuisine ». Qu'est-ce que cela veut dire?

- Qui raconte l'histoire?

- Comment cela se fait-il que la petite fille ressemble à sa famille?

Les interventions complémentaires du chercheur (questions, étayage) sont du même ordre que celles décrites ci-dessus.

\section{NOTES}

1. Brigitte Weninger \& John A. Rowe (2000) : Le Bonnet rouge, Namur, Éd. Nord-Sud.

2. Par exemple: Biquette, La moufle, La petite fourmi qui va à Jérusalem, La plus mignonne des petites souris, L'énorme rutabaga, La belle mouche...

3. Raphaële Frier \& Audrey Pannuti (2011) : La recette de moi, Paris, Naïve.

\section{RÉSUMÉS}

Une pratique actuelle courante au cours préparatoire, et au-delà, dans le cadre des séances de lecture-compréhension, consiste à demander aux élèves de produire des hypothèses sur le sens du texte à partir de premiers indices prélevés sur la couverture, les images de l'album ou un feuilletage. Nous analysons ici ce type de pratiques et ses effets, à partir de l'analyse de deux séquences enregistrées et retranscrites de lecture d'albums, dans une classe de cours préparatoire de zone urbaine sensible. Les corpus ont été constitués dans le cadre d'une étude plus large des rapports entre supports complexes et modes de faire de l'enseignant et des effets différenciateurs de ce rapport selon l'origine sociale et le niveau scolaire des élèves. À l'issue du travail observé au cours des deux séquences, la plupart des élèves de cette classe échoue à construire un modèle mental compatible avec les données de l'album. L'analyse des interactions langagières met au jour l'importance de l'étayage de l'enseignante dans les échanges oraux à propos du texte et permet d'observer la construction d'interprétations erronées par certains 
élèves. À la difficulté d'un support exigeant vient s'ajouter la difficulté à réorganiser par la suite une représentation erronée de l'histoire, élaborée lors des phases d'émission d'hypothèses.

Teachers in France often resort to prediction during reading instruction from first grade onwards. They encourage the students to anticipate the meaning of the text they are about to read, either by examining the cover or by flipping through it. This study examines how this widespread teaching practice influences students' comprehension of complex picture books. This research takes place in a larger study devoted to relationship between complex reading materials, the way teachers use these materials in classrooms and underachievement by disadvantaged students. We recorded and transcribed more than 18 hours of verbal interactions during the class sessions; assessments of the students' reading comprehension of the books was based on a corpus of students' productions. Finally, after reading the books in full and following several discussions of their contents, most of the students failed to construct a mental model consistent with the story. The analysis of oral interactions in the classroom demonstrates how these misunderstandings emerge in the classroom, for example through making predictions, and why they persist in many students' minds, despite the teacher's attempt to clarify some texts' dimensions in comprehension discussions.

INDEX

Keywords : elementary education, literacy, reading comprehension, children's literature Mots-clés : enseignement primaire, didactique, littérature de jeunesse, compréhension, inégalités

\section{AUTEURS}

CAROLINE VIRIOT-GOELDEL

Université Paris-Est, EA 4384 Circeft, Université Paris 8, UPEC, 94010 Créteil Cedex

\section{JACQUES CRINON}

Université Paris-Est, EA 4384 Circeft, Université Paris 8, UPEC, 94010 Créteil Cedex 